Proceedings

\title{
Thermal Stability of Micro-Structured PDMS Piezo-Electrets under Various Polymeric Reticulation Ratios for Sensor Applications ${ }^{\dagger}$
}

\author{
Achraf Kachroudi ${ }^{1, *}$, Skandar Basrour ${ }^{2}$ and Alain Sylvestre ${ }^{1}$ \\ 1 University Grenoble Alpes, CNRS, Grenoble INP, G2Elab, F-38000 Grenoble, France; \\ alain.sylvestre@g2elab.grenoble-inp.fr \\ 2 Institute of Engineering University Grenoble Alpes, University Grenoble Alpes, CNRS, Grenoble INP, \\ TIMA, F-38000 Grenoble, France; skandar.basrour@univ-grenoble-alpes.fr \\ * Correspondence: achraf.kachroudi@g2elab.grenoble-inp.fr \\ + Presented at the Eurosensors 2017 Conference, Paris, France, 3-6 September 2017.
}

Published: 21 August 2017

\begin{abstract}
The effect of the polymeric reticulation on the thermal stability of the piezoelectricity of a micro-structured PDMS piezo-electret was investigated. This new family of flexible piezoelectric materials with very low Young's modulus is used for wearable sensors and energy harvester devices. The best thermal stability was obtained for the piezo-electret material made from the ratio 1:10 between the prepolymer and the crosslinking agent with a longitudinal piezoelectric coefficient $\mathrm{d}_{33}=350 \mathrm{pC} / \mathrm{N}$. The highest piezoelectric coefficient $\mathrm{d}_{33}=750 \mathrm{pC} / \mathrm{N}$ was obtained for the ratio 1:20, but the piezoelectricity was lost at $45^{\circ} \mathrm{C}$ by thermally stimulated discharge.
\end{abstract}

Keywords: piezo-electret; PDMS; electro-active polymer; piezoelectricity; thermal stability

\section{Introduction}

Piezoelectric materials became of great interest since their discovery in the sensors and actuators field. Ceramic materials such as Lead Zirconate Titanate (PZT), Barium Titanate ( $\left.\mathrm{BaTiO}_{3}\right)$, Potassium Niobate $\left(\mathrm{KNbO}_{3}\right)$ and semi-conductors such as Zinc Oxide $(\mathrm{ZnO})$ and Aluminum Nitride $(\mathrm{AlN})$ are widely used in ultrasonic sensors and microsystems devices [1]. However, depending on the targeted applications, we found that other factors than piezoelectricity are invoked such as the acoustic impedance, the shape, the thermal stability and especially the flexibility for wearable and compliant devices applications. For this reason, the piezoelectric ceramic and semi-conductor materials find their field of applications limited. The first alternative proposed to overcome this problem was the use of other materials such as ferroelectric massive polymers (Polyvinylidene Fluoride (PVDF), Polyvinylidene Fluoride/Trifluoroethylene (PVDF-TrFE) [2] and Parylene C) [3]. Nevertheless, the solutions remain partial due to their poor piezoelectric coefficients compared to those of the conventional ceramic and semi-conductor piezoelectric materials. The second alternative proposed was to use piezocomposite materials such as $\mathrm{BaTiO}_{3}-\mathrm{Polydimethylsiloxane} \mathrm{PDMS}$ and $\mathrm{ZnO}-\mathrm{SU} 8$ that consist of introducing ceramic particles into a polymer host matrix in a random or periodic way [2]. The desired effect through these structures was to combine the flexibility of the host polymer matrix and the large piezoelectricity of the ceramic particles. However, the flexibility obtained with such materials was limited. In recent years, another family of piezoelectric polymer materials has appeared and seems to constitute good candidates as a compromise combining flexibility and significant piezoelectric effect [4]. These so-called piezo-electrets are heterogeneous materials exhibiting a piezoelectric behavior with thicknesses varying from few dozen to few hundred microns. They combined in their shape a host matrix containing micro-cavities. To ensure the piezoelectric behavior, these materials are polarized by high voltages, implanting opposite charge carriers in the 
inner surfaces of the micro-cavities and creating macro-dipoles. Typically, these materials are used in their thickness mode [5]. Most piezo-electrets are made by gas injection and extrusion process or by mechanical stretching in order to eliminate injected particles initially introduced in the host polymer matrix for producing the desired cellular structures. The resulting materials are usually ill-controlled in both the form and the shape micro-cavities, and the developed analytical models are not able to predict the dielectric, mechanical and piezoelectric properties. In counterpart, the molding process seem appear as a best way approach by micro-fabrication technique to solve this problem and to be able to control the individual micro-cavity geometry and the overall cellular structure. In this paper, we report a design, micro-fabrication, piezoelectric characterizations and thermal stability of a PDMS piezo-electret material made with various polymeric reticulations for sensor applications.

\section{Experimental Considerations}

According to our previous work concerning the optimization of the piezoelectric response of the PDMS piezo-electret material [6,7], the geometrical parameters of the micro-cavities and the polarization voltage parameters are fixed. The structures are in the form of sandwiches composed of two bulk layers of $55 \mu \mathrm{m}$ thickness separated by a micro-structured $40 \mu \mathrm{m}$ thick layer. The latter is developed by the molding process reported in our previous work [6,7]. The micro-structured layer contains cylindrical micro-cavities of $100 \mu \mathrm{m}$ diameter, $40 \mu \mathrm{m}$ height and distributed regularly in the both surface plan directions with a pitch of $150 \mu \mathrm{m}$. The bulk and micro-structured layers are bonded by an oxygen plasma treatment. Gold electrodes of $100 \mathrm{~nm}$ thickness are sputtered on both samples sides by physical vapor deposition. The structures are polarized by a quasi-static triangular voltage of $0.5 \mathrm{~Hz}$ frequency and with amplitudes between $1 \mathrm{kV}$ and $4 \mathrm{kV}$ for $15 \mathrm{~min}$. The tested piezo-electret materials were with an electrode area of $2 \times 2 \mathrm{~cm}^{2}$.

According to previous works [8], the dielectric spectroscopy is exploited to study the indirect piezoelectric properties of the materials thus obtained. More specifically, dielectric measurements (real and imaginary parts of the piezo-electret material complex capacitance) were carried out in the range $0.1 \mathrm{~Hz}-1 \mathrm{MHz}$ and for temperatures ranging from $-25^{\circ} \mathrm{C}$ to $85^{\circ} \mathrm{C}$. If the frequency of a mechanical resonance mode matches with a frequency of the applied voltage, a resonant peak on the imaginary part of the complex capacitance appears. This peak constitutes the signature of the piezoelectric response of the material. The complex capacitance of this piezoelectric material in thickness mode is given by Equation (1).

$$
C(f)=\frac{\varepsilon_{0} \varepsilon_{r}^{*} A}{h} \frac{1}{1 k_{33}^{* 2} \frac{\tan \left(\pi f / 2 f_{r}^{*}\right)}{\left(\pi f / 2 f_{r}^{*}\right)}}-i C_{\text {loss }}
$$

where $\varepsilon_{0}$ is the free space permittivity, $\varepsilon_{r}^{*}$ is the sample complex relative permittivity, $A$ and $h$ are the area of electrodes and the thickness of the sample respectively. $k_{33}^{*}$ is the complex coupling factor and $f_{r}^{*}$ is the complex anti-resonance frequency of the thickness-extension mode. $C_{\text {loss }}$ constitutes the contribution of the dielectric losses occurring in the material other than those related to the resonance phenomena. $f_{r}^{*}$ is related to the complex elastic stiffness $c_{33}^{*}$ and the mass density $\rho$ through Equation (2).

$$
f_{r}^{*}=\frac{1}{2 h} \sqrt{\frac{c_{33}^{*}}{\rho}}
$$

The coupling factor $k_{33}$ is given by Equation (3).

$$
k_{33}^{2}=\frac{d_{33}^{2} c_{33}}{\varepsilon_{0} \varepsilon_{r}}
$$


$d_{33}, c_{33}$ and $\varepsilon_{r}$ represent the modulus of the longitudinal piezoelectric coefficient, the elastic stiffness and the relative permittivity respectively.

\section{Results and Discussion}

Figure 1 reports only the imaginary part of the complex piezo-electret capacitance (which allows us to study easily the piezoelectric effect) for different polymeric reticulation ratios 1:5, 1:10, 1:15 and 1:20 prepared from the prepolymer and the curing agent of the PDMS kit Sylgard 184 from Dow corning. The isothermal curves are fitted through the imaginary part of Equation (1) based on the Kramers-Kronig relation. The temperature dependence of the determined longitudinal piezoelectric coefficient is presented in Figure 2.

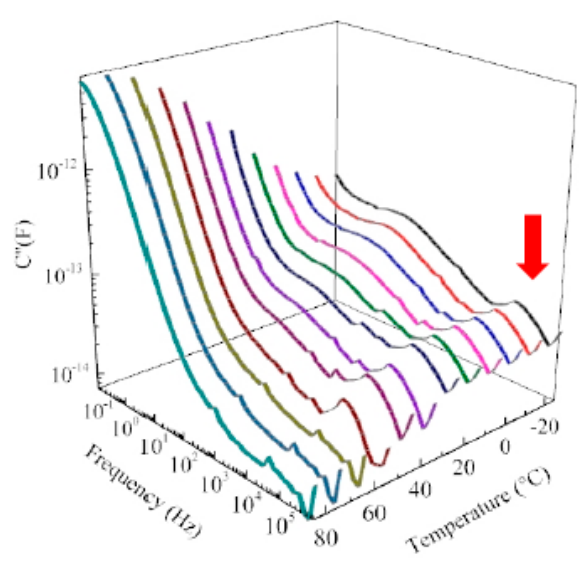

(a)

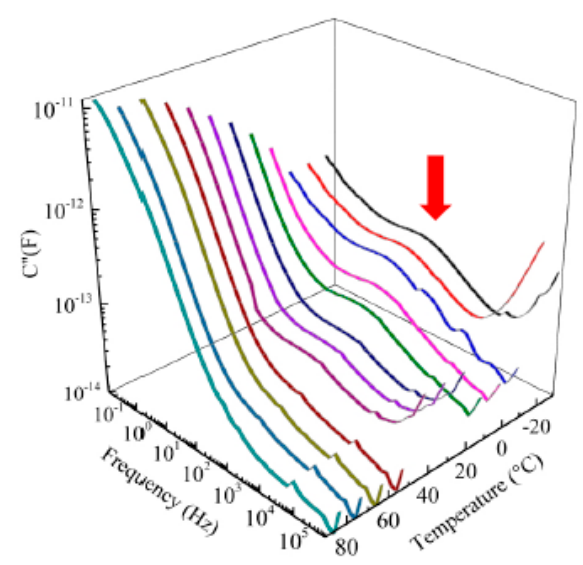

(c)

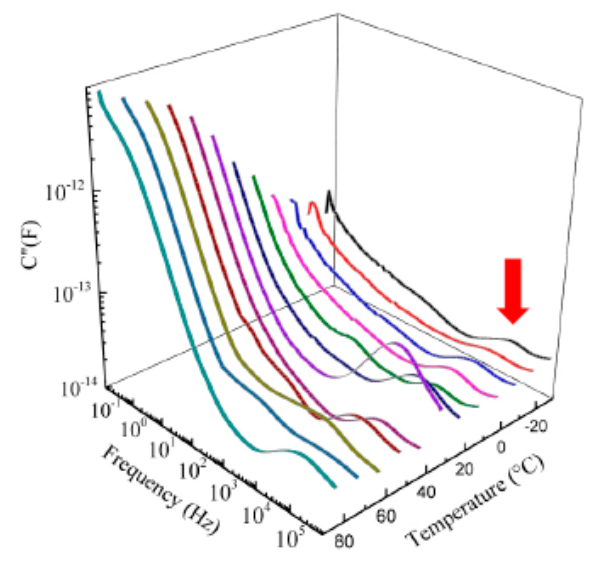

(b)

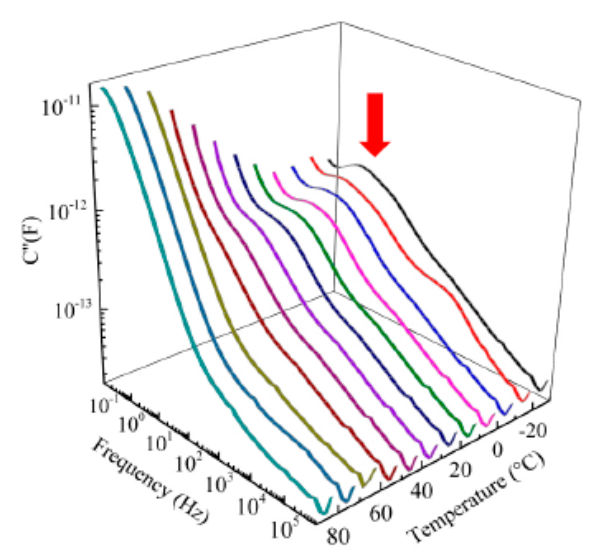

(d)

Figure 1. Frequency-temperature map of the dielectric loss $C^{\prime \prime}$ of the piezo-electret PDMS material with ratios (a) 1:5; (b) 1:10; (c) 1:15 and (d) 1:20. 


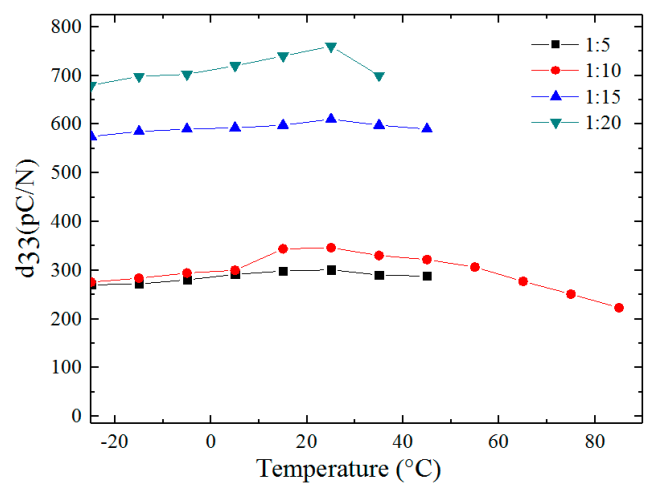

Figure 2. Temperature dependence of the piezoelectric coefficient $\mathrm{d}_{33}$ for PDMS piezo-electrets under various polymeric reticulation.

According to Figure 2, the highest longitudinal piezoelectric coefficient is identified for the 1:20 ratio PDMS piezo-electret material $\left(\mathrm{d}_{33}=750 \mathrm{pC} / \mathrm{N}\right)$ at $25^{\circ} \mathrm{C}$. This is mainly due to the lower Young's modulus of this material as reported in our previous work [4]. However, this ratio presents the lowest thermal stability as the piezoelectricity is lost at $45^{\circ} \mathrm{C}$. This effect is due to the strong deformation of the micro-cavity under high temperature contributing to the reduction of the micro-structured layer thickness and the decrease of the piezoelectricity. Another possible effect is the thermally stimulated discharge of the charge carriers trapped in the micro-cavities inner surfaces. On the other hand, materials made with the ratio 1:10 recommended by the suppliers and several authors exhibit the highest thermal stability with a longitudinal piezoelectric coefficient $\mathrm{d}_{33}=350 \mathrm{pC} / \mathrm{N}$ at $25{ }^{\circ} \mathrm{C}$. This thermal stability is attributed to the fact that this ratio allows the structure to resist to the high deformation of the micro-cavities and to ensure the stability of the implanted charges in the micro-cavities. A compromise should be taken into consideration if a high piezoelectricity is desired (in this case ratio 1:20 is preferred) and if a high thermal stability is chosen (ratio 1:10 is then preferred).

\section{Conclusions and Future Work}

Capabilities in term of piezoelectricity and thermal stability were investigated in PDMS piezo-electret materials. The polymeric reticulation aspect ratio has constituted the main parameter to consider in this study to reach performances. A high longitudinal piezoelectric coefficient of $750 \mathrm{pC} / \mathrm{N}$ is obtained for the materials made of polymeric reticulation ratio of 1:20 and a high thermal stability of the piezoelectricity is obtained for the materials made with the ratio 1:10. For the next step, it will be the implementation of these materials in an energy harvester device.

Conflicts of Interest: The authors declare no conflict of interest.

\section{References}

1. Jaffe, B. Piezoelectric Ceramics; Elsevier: Amsterdam, The Netherlands, 2012; ISBN 978-0-323-15568-7.

2. Ramadan, K.S.; Sameoto, D.; Evoy, S. A review of piezoelectric polymers as functional materials for electromechanical transducers. Smart Mater. Struct. 2014, 23, 33001, doi:10.1088/0964-1726/23/3/033001.

3. Kim, J.Y.H.; Cheng, A.; Tai, Y.C. Parylene-C as a piezoelectric material. In Proceedings of the 2011 IEEE 24th International Conference on Micro Electro Mechanical Systems, Cancun, Mexico, 23-27 January 2011; pp. 473-476.

4. Kachroudi, A.; Basrour, S.; Rufer, L.; Sylvestre, A.; Jomni, F. Micro-structured PDMS piezoelectric enhancement through charging conditions. Smart Mater. Struct. 2016, 25, 105027, doi:10.1088/0964-1726/25/10/105027.

5. Kachroudi, A.; Basrour, S.; Rufer, L.; Jomni, F. Piezoelectric cellular micro-structured PDMS material for micro-sensors and energy harvesting. J. Phys. Conf. Ser. 2015, 660, 12040, doi:10.1088/1742-6596/660/1/012040.

6. Kachroudi, A.; Basrour, S.; Rufer, L.; Sylvestre, A.; Jomni, F. Dielectric properties modelling of cellular structures with PDMS for micro-sensor applications. Smart Mater. Struct. 2015, 24, 125013, doi:10.1088/0964-1726/24/12/125013. 
7. Kachroudi, A.; Basrour, S.; Rufer, L.; Jomni, F. Air-spaced PDMS piezo-electret cantilevers for vibration energy harvesting. J. Phys. Conf. Ser. 2016, 773, 12072, doi:10.1088/1742-6596/773/1/012072.

8. Mellinger, A. Dielectric resonance spectroscopy: A versatile tool in the quest for better piezoelectric polymers. IEEE Trans. Dielectr. Electr. Insul. 2003, 10, 842-861, doi:10.1109/TDEI.2003.1237333.

(C) 2017 by the authors. Licensee MDPI, Basel, Switzerland. This article is an open access article distributed under the terms and conditions of the Creative Commons Attribution (CC BY) license (http://creativecommons.org/licenses/by/4.0/). 